\title{
The Zernike Expansion - An Example of a Merit Function for 2D/3D Registration Based on Orthogonal Functions
}

\author{
Shuo Dong ${ }^{1,2}$, Joachim Kettenbach ${ }^{3}$, Isabella Hinterleitner ${ }^{1}$, \\ Helmar Bergmann ${ }^{1}$, and Wolfgang Birkfellner ${ }^{1}$ \\ ${ }^{1}$ Center of Biomedical Engineering and Physics, Medical University Vienna, Austria \\ wolfgang . birkfellner@meduniwien . ac . at \\ ${ }^{2}$ School of Biomedical Engineering, Capital Medical University, Beijing, P.R. China \\ ${ }^{3}$ University Clinic of Diagnostic Radiology, Department of Interventional Radiology, \\ Medical University Vienna, Austria
}

\begin{abstract}
Current merit functions for 2D/3D registration usually rely on comparing pixels or small regions of images using some sort of statistical measure. Problems connected to this paradigm the sometimes problematic behaviour of the method if noise or artefacts (for instance a guide wire) are present on the projective image. We present a merit function for $2 \mathrm{D} / 3 \mathrm{D}$ registration which utilizes the decomposition of the X-ray and the DRR under comparison into orthogonal Zernike moments; the quality of the match is assessed by an iterative comparison of expansion coefficients. Results in a imaging study on a physical phantom show that - compared to standard cross-correlation - the Zernike moment based merit function shows better robustness if histogram content in images under comparison is different, and that time expenses are comparable if the merit function is constructed out of a few significant moments only.
\end{abstract}

\section{Introduction}

$2 \mathrm{D} / 3 \mathrm{D}$ registration has gained considerable interest in applications for image-guided therapy (IGT) ever since it's introduction to the field of frameless stereotaxy several years ago [1]. Basically, a 6 degree-of-freedom (dof) volume transform is found by iteratively comparing digitally reconstructed radiographs (DRR) derived by volume rendering from computed tomography (CT) volumes 344. A calibrated X-ray device with known projection geometry is used to take an image of the patient, and by solving a variation problem in 6 dof, the registration can be found without having to identify markers or segmenting structures.

Our research effort in this field aims at improving the robustness of $2 \mathrm{D} / 3 \mathrm{D}$ registration by defining a new merit function. In this paper, we present a novel inplane rotation invariant merit function for $2 \mathrm{D} / 3 \mathrm{D}$ registration based on Zernike decompositions of the DRR and the X-ray image to be compared. Advantages include robustness to histogram differences, in-plane rotation invariance (which can be used to simplify the iterative registration), and the possibility to assess the quality of the match since the presented merit function converges to zero in the case of a perfect match. 


\section{Material and Methods}

\subsection{The Zernike Decomposition}

A possibility to construct a merit function for $2 \mathrm{D} / 3 \mathrm{D}$ registration is the projection of the X-ray and DRR images to an orthogonal set of base functions. Subsequent comparison of linear independent series expansion coefficients provides a possibility to assess the match between the two 2D images. One example of such a series expansion is the Zernike expansion. In a Nobel-prize winning paper [5], F. Zernike introduced an orthogonal set of complex polynomials defined on the unit circle. A good introduction to the Zernike decomposition in image processing can be found in [6]. Basically, for every image point $\boldsymbol{q}=(x, y)^{T}$ with $x^{2}+y^{2} \leq 1$, the Zernike polynomials take the form

$$
\mathcal{V}_{n, m}(\boldsymbol{q})=\mathcal{R}_{n, m}(\rho) e^{i m \Theta}
$$

if the image is transformed from cartesian coordinates $(x, y)^{T}$ to polar coordinates $(\rho, \Theta)^{T}$ where the radial polynomials $\mathcal{R}_{n, m}(\rho)$ are given as

$$
\begin{aligned}
\mathcal{R}_{n, m}(\rho)=\sum_{s=0}^{\frac{n-|m|}{2}}(-1)^{s}(n-s) ! \frac{\rho^{n-2 s}}{s !\left(\left(\frac{n+|m|}{2}\right)-s\right) !\left(\left(\frac{n-|m|}{2}\right)-s\right) !} & \\
n \ldots & \text { Integer, } n \geq 0 . \\
m & . \text { Integer, } \bmod (n-|m|, 2)=0, \\
& \quad \text { and }|m| \leq n . \\
\rho \ldots & \text { Distance of pixel } \boldsymbol{q} \text { to image } \\
& \quad \text { center, } \rho=\|\boldsymbol{q}\| . \\
\Theta \ldots & \text { Angle between } \boldsymbol{q} \text { and x-axis, } \\
& \text { counterclockwise. } \\
i \ldots & \text { imaginary unit }
\end{aligned}
$$

Denote that in the case of the radial polynomials as given in eq. 2 the identity

$$
\mathcal{R}_{n,-m}(\rho)=\mathcal{R}_{n, m}(\rho)
$$

is fulfilled. The functions $\mathcal{V}_{n, m}$ are orthogonal, thus fulfilling

$$
\iint_{x^{2}+y^{2} \leq 1} \mathcal{V}_{n, m}^{*}(\boldsymbol{q}) \mathcal{V}_{p, q}(\boldsymbol{q}) \mathrm{d} x \mathrm{~d} y=\frac{\pi}{n+1} \delta_{n, p} \delta_{m, q}
$$

with $\delta_{i, j}$ being the Kronecker function, which is 1 if $i=j$ and 0 otherwise. Projections of the image to these base functions $\mathcal{V}$ give linear independent coefficients, the so-called Zernike moments, and the image can be rewritten as a series expansion. For a discrete digital image $I(\boldsymbol{q})$, the coefficients are defined as

$$
A_{n, m}=\frac{n+1}{\pi} \sum_{x} \sum_{y} I(\boldsymbol{q}) \mathcal{V}_{n, m}^{*}(\rho, \Theta)
$$




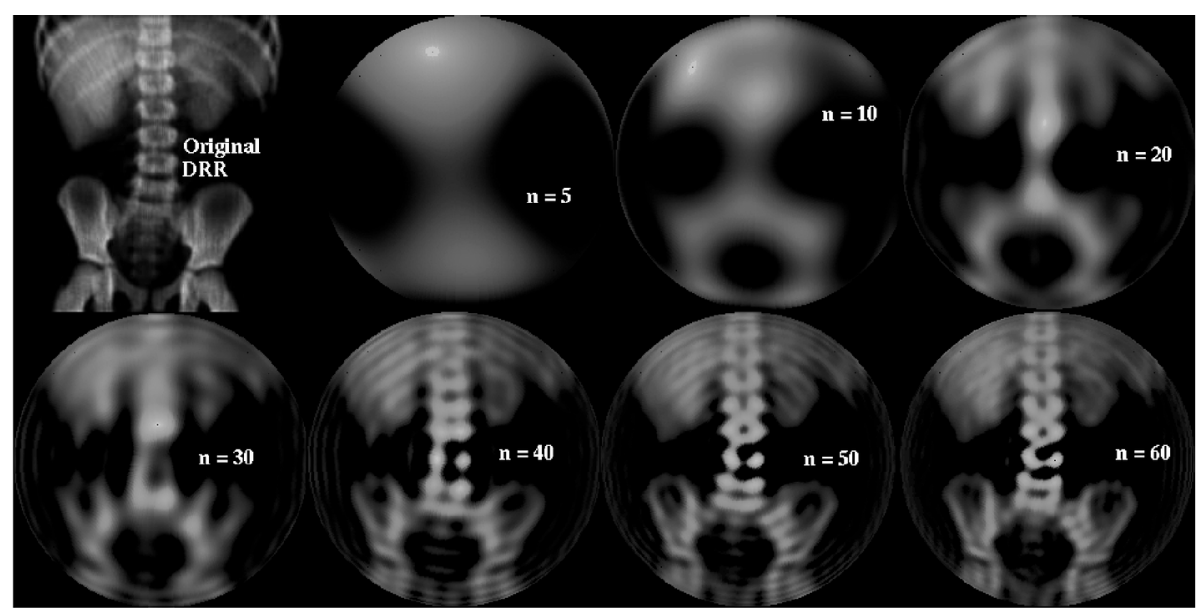

Fig. 1. Zernike reconstruction of a DRR for varying orders $n$. Denote the small change in detail for higher orders. Another remarkable detail is the presence of ringing artefacts close to the circular image borders - this is a general phenomenon for series expansions in the vicinity of discontinuities.

with $x^{2}+y^{2} \leq 1$. $\mathcal{V}_{n, m}^{*}(\rho, \Theta)$ is the complex conjugate of $\mathcal{V}_{n, m}(\rho, \Theta)$. It can easily be verified that

$$
A_{n, m}^{*}=A_{n,-m}
$$

Reconstruction of an image $\hat{I}(\boldsymbol{q})$ from it's coefficients is simple; actually, one has to compute

$$
\hat{I}(\boldsymbol{q})=\sum_{n=0}^{N_{\max }} \sum_{m} A_{n, m} \mathcal{V}_{n, m}(\boldsymbol{q})
$$

An illustration of $\hat{I}$ for varying levels of $N_{\max }$ is given in fig. 1]

In [7/8, a more efficient recursive method for computing Zernike base functions is given:

$$
\begin{aligned}
\mathcal{R}_{(n+2), m}(\rho)=\frac{1}{K_{1}} & \left(\left(K_{2} \rho^{2}+K_{3}\right) \mathcal{R}_{n, m}(\rho)+K_{4} \mathcal{R}_{(n-2), m}(\rho)\right) \\
K_{1} & =\frac{1}{2}((n+1)(n-1)(n-2)) \\
K_{2} & =2 n(n-1)(n-2) \\
K_{3} & =-(n-1)^{3} \\
K_{4} & =-\frac{1}{2}(n(n-1)(n-3)) .
\end{aligned}
$$

Since this relation is only defined for $m \leq n-2$, the identities

$$
\mathcal{R}_{(n+2),(n+2)}(\rho)=\rho^{n+2}
$$


and

$$
\mathcal{R}_{(n+2), n}(\rho)=(n+2) \rho^{n+2}-(n+1) \rho^{n}
$$

have to be derived from eq. 2, Other than in eq. 2, the effort in computing the base functions $\mathcal{R}_{n, m}$ is significantly reduced here since in eqs. 8, 9], and 10 no faculties have to be computed.

It is easy to verify from eq. 1 that the coefficients $A_{n, m}$ have very simple rotational properties since each rotation of the image by an angle $\alpha$ results in a phase shift of the coefficients; if $A_{n, m}^{\prime}$ are the coefficients of the image $I(\boldsymbol{q})^{\prime}$, which was derived from $I(\boldsymbol{q})$ by rotation of $\alpha$, they are related to the coefficients $A_{n, m}$ of the original image $I(\boldsymbol{q})$ by $A_{n, m}^{\prime}=A_{n, m} e^{-i m \alpha}$. Therefore the identity

$$
\left\|A_{n, m}^{\prime}\right\|=A_{n, m} A_{n, m}^{*}=\left\|A_{n, m}\right\|
$$

holds, and thus the norm of the Zernike coefficients is actually an in-plane rotation-invariant measure. From eq. 6 it is evident that only the coefficients with $m \geq 0$ are relevant in this case.

\subsection{An In-Plane Rotation Invariant Cost Function Based on Zernike Moments for 2D/3D Registration}

For the purpose of comparing images in $2 \mathrm{D} / 3 \mathrm{D}$ registration, a merit function for registration based on Zernike moments can be defined as a comparison of expansion coefficients since the base functions $\mathcal{V}_{n, m}(\boldsymbol{q})$ are orthogonal. Invariance to in-plane rotation is introduced by comparing the norms of the Zernike moments $A_{n, m}$ since the $2 \mathrm{D}$ image rotation is given by the complex phase $\alpha$ of the coefficients. In general, the merit function $Z$ to be minimized takes the form

$$
Z\left(I_{X-\text { ray }}, I_{D R R}\right)=\sum_{n=0}^{N_{\max }} \sum_{m}\left|\left\|A_{n, m}\left(I_{X-\text { ray }}\right)\right\|-\left\|A_{n, m}\left(I_{D R R}\right)\right\|\right| .
$$

A merit function of this type has several characteristics, among which are

- invariance to in-plane image rotation 9 .

- non-negativeness; in case of a perfect match, $Z\left(I_{X-\text { ray }}, I_{D R R}\right)$ converges to zero.

- the level of detail for image comparison is governed by $N_{\max }$.

However, it is evident that computing all Zernike moments is a time-intensive task; depending of the actual value of $N_{\max }$, several hundreds of projections of the image to the base functions $\mathcal{V}_{n, m}(\boldsymbol{q})$ have to be computed. Therefore it is clear that the number of moments to be evaluated has to be constrained if time expenses for image comparison are to be restricted. In particular, the merit function $Z$ was implemented in such a manner that

- $I_{X-\text { ray }}$ and $I_{D R R}$ are dithered to eight bit grayscale images and interpolated to images of equal resolution. These images are stored in polar coordinates $\boldsymbol{q}$. 


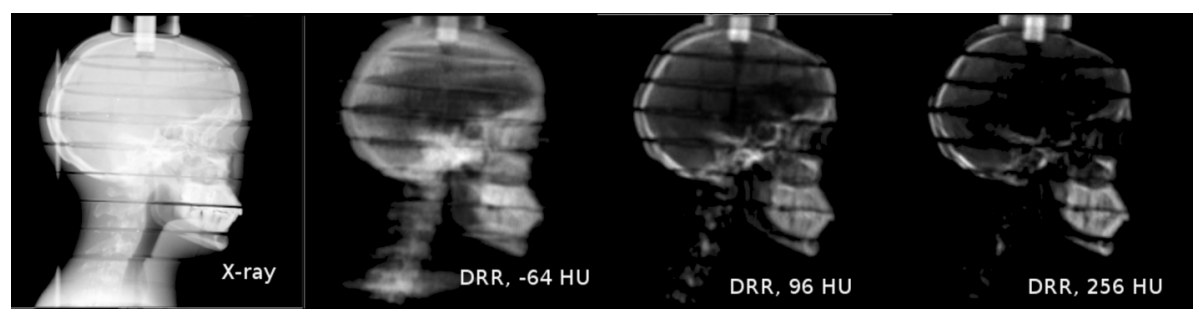

Fig. 2. The image data used for comparing the Zernike merit function to a standard cross-correlation merit function. A conebeam CT of an Alderson-Rando phantom using a LINAC mounted digital x-ray imaging unit was taken and serves as the base dataset for the DRRs. The x-ray was taken using the same machine. Three different minimum thresholds were used for DRR rendering.

- All base functions $\mathcal{V}_{n, m}(\boldsymbol{q})$ are computed up to $N_{\max }$ and stored in lookuptables of a resolution matching the size of $I_{X-\text { ray }}$ and $I_{D R R}$.

- All Zernike moment norms $\left\|A_{n, m}\right\|$ with $n \geq 2$ and $m \geq 2$ were computed for $I_{X-\text { ray }}$ and stored in a $N_{\max } \times 3$ array $H$. Zernike moments with order $n$ or repetition $m$ equal zero or one do usually not contain significant morphological information, whereas their norms tend to be numerically large. Therefore these moments are excluded.

- A sorting algorithm is employed to sort the hashtable $H$ in dependence of $\left\|A_{n, m}\right\|$.

- A given number $o$ of orders $n$ and repetitions $m$ yielding the numerically largest moment norms $\left\|A_{n, m}\right\|$ is selected from $H$. These values of $n$ and $m$ are stored in a $o \times 2$ matrix $H^{\prime}$.

- In the iterative registration process, five dof $\Phi_{x}, \Phi_{y}$, and $\boldsymbol{T}$ are varied; a $4 \times 4$ registration matrix $V$ applied to the CT-volume for rendering $I_{D R R}$ is derived. The $o$ Zernike moments stored in matrix $H^{\prime}$ are computed for $I_{D R R}$. The iterative optimization process stops once $Z\left(I_{X-\text { ray }}, I_{D R R}\right)$ reaches a minimum.

- The decoupled dof $\Phi_{z}$ is computed independently using a standard registration algorithm based on a simple cross-correlation method for the only remaining dof in rotation, and an optimum registration matrix $V^{*}$ containing all six dofs is computed.

- The inverse matrix $V^{*-1}$ as found by the optimization process is applied to the volume.

In this initial evaluation, we have constrained ourselves to merit functions constructed out of $o=3,5$, and 10 coefficents, which keeps the time expenses for registration on a reasonable level.

\subsection{Algorithm Implementation and Evaluation}

For initial testing of the Zernike moment based merit functions, we developed a software called ZERNIKE under SuSE Linux 9.1 (Novell Inc. Waltham/MA) 
using gcc 3.3.1 (Free Software Foundation, Boston/MA) and the Qt 3.1 toolkit (Trolltech, Norway). Basic medical image processing functionality was added using the AVW 8.1 toolkit (Biomedical Imaging Resource, Rochester/MN). Besides the merit function presented, cross-correlation - another typical merit function for $2 \mathrm{D} / 3 \mathrm{D}$ registration - 12 ) was implemented. The GSL-implementations of the Simplex algorithm (Free Software Foundation, Boston/MA) was used for optimization. A DRR computed from a conebeam CT scan of an Alderson-Rando Phantom acquired on an Elekta Synergy LINAC was compared to a calibrated $\mathrm{X}$-ray from the same machine. This setup is not equivalent to a full scale study on patient data, but the anthropomorphic phantom which contains both bony tissue as well as soft tissue equivalent is considered a good approximation for the purpose of algorithm validation. Different minimum thresholds for DRR-generation (-64, 96, and $256 \mathrm{HU}$ ) were used (see also Fig. 2). The algorithm used for DRR generation was wobbled splatting [1011]. Fifteen registrations with a random variation of $\pm 3^{\circ} / 5$ pixels, $\pm 5^{\circ} / 10$ pixels, and $\pm 10^{\circ} / 20$ pixels compared to the approximate initial guess were carried out. The average deviation from the initial guess for all three dof in rotation $\Delta \Phi\left[^{\circ}\right]$ as well as the geometric error in-plane $(\Delta x-y[\mathrm{pix}])$ and out-of-plane $(\Delta z)$ [pix] was recorded.

\section{$3 \quad$ Results}

Detailed results can be found in Table 1. Typical execution time for the registration was approximately 30 seconds for cross-correlation and 40 - 50 seconds for the Zernike- moment merit function. Here, the number of siginificant moments used determines the execution time of the algorithm. Compared to crosscorrelation, the Zernike merit function is less dependent on similar histogram content and delivers more stable results compared to cross - correlation. Another interesting property is the fact that overall scatter in registration results is not strongly influenced by the number of moments used. The simplified selection criterion (which is given in 2.1) for the Zernike moments used leads to the strong use of moments with low repetion $m$. It can therefore be explained that translation results are sometimes better in the case of cross correlation (since fine features are generally omitted), and that an increase in the number of moments used does not necessarily improve registration results.

\section{Discussion}

The initial results given in this paper cannot provide a full evaluation of this novel paradigm in $2 \mathrm{D} / 3 \mathrm{D}$ registeration. From the very nature of the Zernike expansion, it is evident that outliers such as high-intensity artefacts or linear intensity transforms do not affect the merit function to a high level, and this conclusion is also supported by our results. A possible advantage of the Zernike merit function is its invariance to in-plane rotations since only the real norm of the complex coefficent is evaluated; in a setup as proposed in 9 where one dof of rotation is decoupled in a special imaging geometry, this property can be utilized for reducing 
Table 1. Registration results on a physical Alderson-Rando phantom. For a comparison, results with a standard cross-correlation merit function are also presented. Deviations from the initial guess are averaged for fifteen registration runs using a random displacement of the initial guess (indicated as 'Displacement Range' in the table). Varying minimum thresholds for DRR rendering were used, and no histogram equalization was carried out. Results for the Zernike-type merit function are given when using the most significant non-trivial 3, 5, and 10 moments. The best results in each row are typeset in boldface. Compared to cross-correlation, the Zernike merit function is more stable compared to cross-correlation despite the translation results which are sometimes better. However, the cross-correlation merit function is highly apparently affected by the differences in histogram content of the DRRs relative to the X-ray.

\begin{tabular}{|c|c|c|c|c|c|c|}
\hline \multirow{2}{*}{\begin{tabular}{|c|} 
Initial Dis- \\
placement \\
Range
\end{tabular}} & \multirow{2}{*}{\begin{tabular}{|c|} 
Minimum \\
DRR Thres- \\
hold [HU]
\end{tabular}} & \multirow{2}{*}{$\begin{array}{c}\text { Result } \\
\text { Deviations }\end{array}$} & \multicolumn{3}{|c|}{ No. of Zernike Moments } & \multirow{2}{*}{$\begin{array}{l}\text { Cross - } \\
\text { Cor- } \\
\text { relation }\end{array}$} \\
\hline & & & 3 & 5 & 10 & \\
\hline \multirow{9}{*}{$\begin{array}{c}3^{\circ}- \\
5 \text { pixels }\end{array}$} & \multirow[t]{3}{*}{-64} & $\Delta \Phi\left[^{\circ}\right]$ & $3.3 \pm 2.5$ & $2.5 \pm 2.4$ & $4.2 \pm 1.9$ & $13.2 \pm 4.0$ \\
\hline & & $\Delta x-y$ pix & $8.3 \pm 2.9$ & $10.8 \pm 4.2$ & $7.7 \pm 4.3$ & $7.8 \pm 1.5$ \\
\hline & & $\Delta z[\mathrm{pix}]$ & $11.7 \pm 1.1$ & $11.6 \pm 1.6$ & $10.6 \pm 2.0$ & $11.9 \pm 9.5$ \\
\hline & \multirow[t]{3}{*}{96} & $\left.\Delta \Phi{ }^{0}\right]$ & $2.4 \pm 1.1$ & $1.9 \pm 1.0$ & $2.1 \pm 0.9$ & $4.6 \pm 3.7$ \\
\hline & & $\Delta x-y[\mathrm{pix}$ & $7.2 \pm 2.3$ & $6.3 \pm 2.7$ & $7.1 \pm 3.1$ & $2.9 \pm 1.3$ \\
\hline & & $\Delta z[\mathrm{pix}]$ & $8.1 \pm 2.7$ & $8.1 \pm 3.3$ & $7.3 \pm 4.0$ & $2.0 \pm 1.9$ \\
\hline & \multirow[t]{3}{*}{256} & $\Delta \Phi\left[{ }^{0}\right]$ & $2.4 \pm 0.9$ & $2.4 \pm 0.9$ & $2.3 \pm 0.9$ & $6.1 \pm 3.7$ \\
\hline & & $\Delta x-y[\mathrm{pix}]$ & $6.0 \pm 3.0$ & $5.9 \pm 2.3$ & $6.0 \pm 2.9$ & $2.6 \pm 1.3$ \\
\hline & & $\Delta z[\mathrm{pix}]$ & $5.9 \pm 1.4$ & $5.6 \pm 2.0$ & $5.6 \pm 1.9$ & $2.3 \pm 2.3$ \\
\hline \multirow{9}{*}{$\begin{array}{c}5^{\circ}- \\
10 \text { pixels }\end{array}$} & \multirow[t]{3}{*}{-64} & $\Delta \Phi\left[{ }^{\circ}\right]$ & $4.1 \pm 2.3$ & $3.8 \pm 2.9$ & $3.8 \pm 2.6$ & $\overline{12.6 \pm 4.1}$ \\
\hline & & $\Delta x-y$ pix & $10.4 \pm 5.6$ & \begin{tabular}{|l|}
$10.8 \pm 4.0$ \\
\end{tabular} & $10.7 \pm 3.2$ & $7.7 \pm 5.7$ \\
\hline & & $\Delta z[$ pix $]$ & $10.7 \pm 2.5$ & $10.2 \pm 3.3$ & $12.1 \pm 2.1$ & $12.1 \pm 7.1$ \\
\hline & \multirow[t]{3}{*}{96} & $\left.\Delta \Phi{ }^{\circ}\right]$ & $3.4 \pm 2.1$ & \begin{tabular}{|l|}
$3.3 \pm 2.0$ \\
\end{tabular} & $3.8 \pm 2.1$ & $15.6 \pm 8.1$ \\
\hline & & $\Delta x-y[$ pix & $8.1 \pm 3.8$ & $7.2 \pm 3.5$ & $7.5 \pm 3.5$ & $8.3 \pm 5.8$ \\
\hline & & $\Delta z[\mathrm{pix}]$ & $8.3 \pm 3.6$ & $8.1 \pm 3.1$ & $9.1 \pm 4.1$ & $13.8 \pm 10.2$ \\
\hline & \multirow[t]{3}{*}{256} & $\Delta \Phi[0]$ & $3.2 \pm 1.3$ & $3.1 \pm 1.3$ & $2.7 \pm 1.7$ & $24.9 \pm 21.9$ \\
\hline & & $\Delta x-y[\mathrm{pix}$ & $7.1 \pm 3.1$ & $6.4 \pm 3.4$ & $6.6 \pm 3.7$ & $20.1 \pm 9.4$ \\
\hline & & $\Delta z[\mathrm{pix}]$ & $6.7 \pm 1.9$ & $6.7 \pm 2.0$ & $5.7 \pm 1.8$ & $28.0 \pm 20.7$ \\
\hline \multirow{9}{*}{$\begin{array}{c}10^{\circ}- \\
20 \text { pixels }\end{array}$} & \multirow[t]{3}{*}{-64} & $\Delta \Phi\left[^{\circ}\right]$ & $4.2 \pm 2.9$ & $6.1 \pm 4.6$ & $8.2 \pm 5.5$ & $12.9 \pm 4.4$ \\
\hline & & $\Delta x-y[$ pix & $14.5 \pm 6.0$ & $17.0 \pm 9.5$ & $18.4 \pm 11.2$ & $13.6 \pm 8.6$ \\
\hline & & $\Delta z[\operatorname{pix}]$ & $12.4 \pm 6.4$ & $13.3 \pm 6.5$ & $15.2 \pm 7.8$ & $11.5 \pm 8.3$ \\
\hline & \multirow[t]{3}{*}{96} & $\Delta \Phi[0]$ & $6.5 \pm 3.4$ & $6.2 \pm 2.6$ & $8.3 \pm 4.1$ & $16.6 \pm 9.1$ \\
\hline & & $\Delta x-y$ pix & $15.9 \pm 9.0$ & $16.7 \pm 7.3$ & $18.5 \pm 10.6$ & $9.6 \pm 6.3$ \\
\hline & & $\Delta z[\mathrm{pix}]$ & $11.7 \pm 8.0$ & $13.4 \pm 6.7$ & $16.7 \pm 10.7$ & $21.2 \pm 15.6$ \\
\hline & \multirow[t]{3}{*}{256} & $\Delta \Phi\left[^{\circ}\right]$ & $6.5 \pm 5.3$ & $5.1 \pm 3.4$ & $4.8 \pm 2.7$ & $23.7 \pm 15.2$ \\
\hline & & $\Delta x-y[\mathrm{pix}$ & $18.3 \pm 6.0$ & $13.4 \pm 9.3$ & $20.2 \pm 7.2$ & $14.5 \pm 12.3$ \\
\hline & & $\Delta z[\mathrm{pix}]$ & $18.1 \pm 20.5$ & $15.9 \pm 23.1$ & $7.4 \pm 3.1$ & $17.1 \pm 12.2$ \\
\hline
\end{tabular}

the computational effort in the iterative registration process. Another advantage lies in the fact that fine image detail does usually not affect low-order coefficients, while the coefficient itself always is non-ambigous due to the orthonormality of the base functional system. The reason lies in the fact that a merit function based on series expansions exploits geometric image features rather than a functional relationship between pixels. The Zernike moments should therefore outperform other approaches to image simplification such as downscaling and low-pass filtering; the improved robustness of the Zernike merit function compared to the straightforward cross-correlation approach which is evident from the results supports this assumption. These aspects are, however, subject to further research and cannot be answered completely within the limitations of this paper. Another interesting application of Zernike series could, however, be image-based content retrieval: a 
situation where a similar X-ray, which represents the best match for a given CTscan, is to be found, a 2D/3D registration of the CT to a set of pre-selected X-ray images with pre-computed Zernike representations would provide a possibility to accomplish this task. Within view of this application, another interesting property of the Zernike-decomposition comes into play. For a perfect match, eq. 12 is zero. Therefore, the residual value of eq. 12 can be used as an overall measure of the match found in the database.

\section{Acknowledgments}

Analyze AVW was supplied by Dr. R. A. Robb, Biomedical Imaging Ressource, Mayo Clinic, Rochester/MN. This work was supported by the Austrian Science Foundation FWF under grants P19931 and TRP L503. S. Dong was also supported by the ASEA-Uninet foundation.

\section{References}

1. Lemieux, L., Jagoe, R., Fish, D.R., Kitchen, N.D., Thomas, D.: A patient-tocomputed-tomography image registration method based on digitally reconstructed radiographs. Med. Phys. 21(11), 1749-1760 (1994)

2. Penney, G.P., Weese, J., Little, J.A., Desmedt, P., Hill, D.L., Hawkes, D.: A comparison of similarity measures for use in $2-\mathrm{D}-3-\mathrm{D}$ medical image registration. IEEE Trans. Med. Imaging 17(4), 586-595 (1998)

3. Tomazevic, D., Likar, B., Pernus, F.: 3D/2D image registration: the impact of X-ray views and their number. MICCAI 10 (Pt 1), 450-457 (2007)

4. Birkfellner, W., Figl, M., Kettenbach, J., Hummel, J., Homolka, P., Schernthaner, R., Nau, T., Bergmann, H.: Rigid 2D/3D slice-to-volume registration and its application on fluoroscopic CT images. Med. Phys. 34(1), 246-255 (2007)

5. Zernike, F.: Beugungstheorie des Schneidenverfahrens und seiner verbesserten Form, der Phasenkontrastmethode

6. Khotanzad, A., Hong, Y.: Invariant image recognition by Zernike moments. IEEE Trans. Pattern Anal. Mach. Intell. 12(5), 489-497 (1990)

7. Kintner, E.: On the mathematical properties of Zernike polynomials. Optica Acta 23(8), 679-680 (1976)

8. Chong, C.W., Raveendran, P., Mukundian, R.: A comparative analysis of algorithms for fast computation of Zernike moments. Pattern Recognition 36(3), 731$742(2003)$

9. Birkfellner, W., Wirth, J., Burgstaller, W., Baumann, B., Staedele, H., Hammer, B., Gellrich, N.C., Jacob, A.L., Regazzoni, P., Messmer, P.: A faster method for 3D/2D medical image registration-a simulation study. Phys. Med. Biol. 48(16), 2665-2679 (2003)

10. Birkfellner, W., Seemann, R., Figl, M., Hummel, J., Ede, C., Homolka, P., Yang, X., Niederer, P., Bergmann, H.: Wobbled splatting - a fast perspective volume rendering method for simulation of x-ray images from CT. Phys. Med. Biol. 50(9), N73-84(2005)

11. Spoerk, J., Bergmann, H., Wanschitz, F., Dong, S., Birkfellner, W.: Fast DRR splat rendering using common consumer graphics hardware. Med. Phys. 34(11), 4302-4308 (2007) 